\title{
Localised prostate cancer and hemophilia A (AHA): Case report and management of the disease
}

\author{
Francesco Celestino $^{1}$, Cristian Verri ${ }^{2}$, Francesco De Carlo ${ }^{2}$, Savino Mauro Di Stasi ${ }^{2}$ \\ ${ }^{1}$ Urologic Oncology Unit, Policlinico Casilino, Rome, Italy; \\ 2 Department of Experimental Medicine and Surgery, Tor Vergata University, Rome, Italy.
}

\begin{abstract}
Summary Acquired Hemophilia A (AHA) is a rare bleeding diathesis characterized by the development of autoantibodies against factor VIII (FVIII). About half of the cases are idiopathic and the other half are associated with autoimmune diseases, postpartum problems, infections, inflammatory bowel disease, drugs, lymphoproliferative disorders or solid tumors. AHA is associated with malignancies in $7-15 \%$ of cases. We report a case of AHA in a 65 year old patient with prostatic carcinoma, who underwent retropubic radical prostatectomy (RP).
\end{abstract}

KEY WORDS: Activated partial thromboplastin time; Factor VIII; Hemophilia A; Prostate cancer; Radical prostatectomy.

Submitted 14 July 2014; Accepted 1 August 2014

\section{INTRODUCTION}

AHA is a bleeding disorder in which autoantibodies, usually IgG, are produced against Factor VIII (FVIII). The clinical presentation varies from light bleeding to acute and life-threatening hemorrhages. The mortality rate ranges from 7.9 to $22 \%(1,2)$.

\section{Case report}

A 65-year-old Caucasian male, with no significant medical history, underwent retropubic radical prostatectomy (RP) for prostatic carcinoma.

His PSA was $5.2 \mathrm{ng} / \mathrm{ml}$, Hb was $14.6 \mathrm{~g} / \mathrm{dl}$. Coagulation studies revealed an elevated activated partial thromboplastin time (aPTT) of 37.4 seconds $(20.0-35.0 \mathrm{sec}$.) and normal prothrombin time (PT). In the immediate postoperative period, $\mathrm{Hb}$ was $10.7 \mathrm{~g} / \mathrm{dl}$. On $7^{\text {th }}$ postoperative day, $\mathrm{Hb}$ was $6.7 \mathrm{~g} / \mathrm{dl}$, the urine was clear and surgical drainage showed no significant traces, whereas the patient had a CT scan showing active bleeding in the prostate bed anterior to the rectum. The patient underwent surgical revision that showed no major bleeding. On the $11^{\text {th }}$ postoperative day, massive hematuria was noted in the patient. The endoscopic revision showed multiple and small hemorrhagic areas at the bladder neck and posterior bladder wall, which were coagulated.
The aPTT was 61.0 seconds. Two days later there was another massive hematuria, for which the patient underwent selective angiography that showed spreading of the contrast medium from a very thin and tortuous branch of the vesical artery. After several failed attempts at selective cannulation of the vesical artery branch, the patient underwent surgical revision. Ten days later, following another massive hematuria, the patient underwent endoscopic revision. On the $34^{\text {th }}$ postoperative day, he was diagnosed with autoantibodies against FVIII (6 U Bethesda; normal range $0.55 \mathrm{U}$ Bethesda) by a specialized hematological laboratory. The FVIII assay was 20\% (normal range 60-140\%). Initial management was aimed at controlling acute bleeding and then eradicating the inhibitor. Recombinant factor VIII (rFVIIa, NovoSeven ${ }^{\circledR}$, Novo Nordisk, Bagsvaerd, Denmark) was adopted as a primary agent, using the dose of $90 \mu \mathrm{g} / \mathrm{kg}$ every 4 hours when major bleeding occurred and prior to planned invasive procedures. Immunosuppression was initiated with $1 \mathrm{mg} / \mathrm{kg}$ of prednisone daily. Six weeks after immunosuppressive therapy, FVIII autoantibodies were measured at $<0.55 \mathrm{U}$ Bethesda.

\section{Discussion}

AHA is a bleeding disorder in which autoantibodies, usually $\operatorname{IgG}$, are produced against FVIII. Its incidence ranges from 0.2-4 cases/million/year, but this figure may be underestimated, given the difficulty in achieving its diagnosis.

When preoperative coagulation studies reveal an elevated aPTT and normal PT, AHA should be suspected. Some patients may show a normal aPTT and no bleeding during and immediately after surgery. In this cases, the development of FVIII inhibitors can be viewed as a paraneoplastic phenomenon.

AHA is confirmed by detecting the FVIII inhibitor in laboratory tests, but these tests are available only at specialized hematological laboratories. This lengthens the time needed for diagnosis, resulting in a delay in administering the appropriate anti-hemorrhagic treatment. Therefore, the patient often undergoes surgical or invasive diagnostic procedures that can cause complications requiring blood transfusions. 
Management principles entail controlling bleeding and then eradicating autoantibodies through immunosuppression using a combination of steroids, immunoglobulins, cyclophosphamide and monoclonal antibodies (Table 1). The treatment of acute bleeding episodes involves the use of different bypassing agents, such as recombinant activated factor VII (rFVIIa), activated prothrombin complex concentrate (aPCC, FEIBA ${ }^{\circledR}$ ), desmopressin and human or porcine FVIII concentrates.

Fresh frozen plasma is often ineffective, because it contains a low concentration of FVIII that is quickly inactivated by antibodies.

In patients with a low FVIII antibody titer, desmopressin (Minirin ${ }^{\circledR}$, Ferring, Sweden), synthetic vasopressin analogues can be used to stop bleeding. This is due to the ability of desmopressin to release FVIII from the vascular endothelium to the circulatory system.
There is no optimal regimen for inhibitor eradication. The most common strategy is to use steroids alone or steroids in combination with cyclophosphamide. The average time to remission with steroids is about 5 weeks.

\section{References}

1. Franchini M, Gandini G, Di Paolantonio T, Mariani G. Acquired Hemophilia A: a Concise Review American Journal of Hematology. 2005; 80:55-63.

2. Shetty S, Bhave M, Ghosh K. Acquired hemophilia a: diagnosis, aetiology, clinical spectrum and treatment options Autoimmun Rev. 2011; 10:311-6.

3. Reitter S, Knoebl P, Pabinger I, Lechner K. Postoperative paraneoplastic factor VIII auto-antibodies in patients with solid tumours. Haemophilia. 2011; 17:e889-94.

\section{Table 1.}

Algorithm of acquired hemophilia A patient management (rFVIla - recombinant activated Factor VII (Novoseven ${ }^{\circledR}$ ); aPCC - activated Prothrombin Complex Concentrate; vWF - von Willebrand Factor).

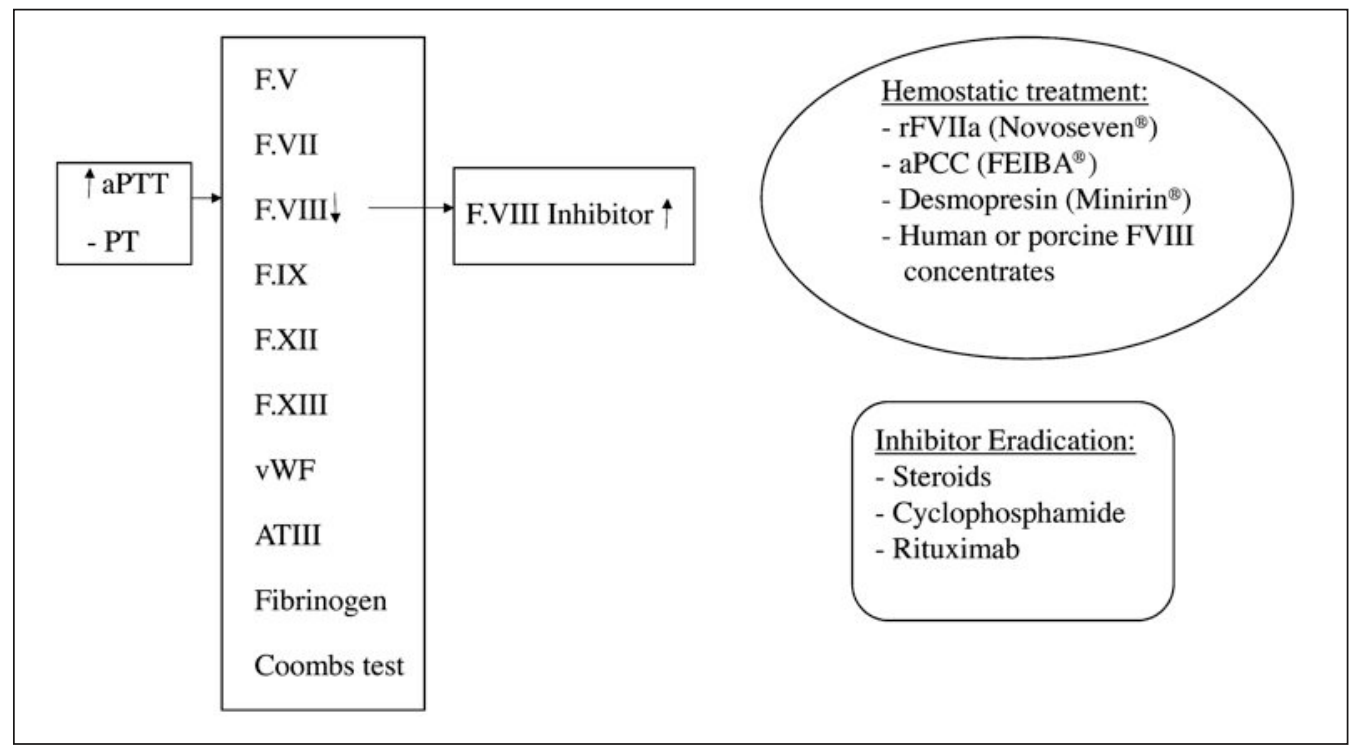

\section{Correspondence}

Francesco Celestino, MD (Corresponding Author)

frapichi@libero.it

Policlinico Casilino

Via Casilina, 1049 - 00169 Rome, Italy

Home: Via G. Rossini, 26 - 81040 Curti (CE), Italy

Cristian Verri, MD

verricris@tiscali.it

Francesco De Carlo, MD

francescodecarlo03@yahoo.it

Savino Mauro Di Stasi, MD

sdistas@tin.it

Department of Experimental Medicine and Surgery

Tor Vergata University, Rome, Italy 